\title{
Enhanced Ant Colony System for Reducing Packet Loss in Wireless Sensor Network
}

\author{
Husna Jamal Abdul Nasir ${ }^{1}$, Ku Ruhana Ku-Mahamud ${ }^{2}$ and Eiji Kamioka ${ }^{3}$ \\ ${ }^{1}$ Faculty of Engineering Technology, Universiti Malaysia Perlis, \\ 02100 Padang Besar, Perlis, Malaysia \\ ${ }^{2}$ School of Computing, College of Arts and Science, Universiti Utara Malaysia, \\ 06010 Sintok, Kedah, Malaysia \\ ${ }^{3}$ Graduate School of Engineering and Science, Shibaura Institute of Technology, \\ Tokyo 135-8548, Japan \\ ${ }^{1}$ husna.jamanas@gmail.com, ${ }^{2}$ ruhana@uum.edu.my, ${ }^{3}$ kamioka@shibaura-it.ac.jp
}

\begin{abstract}
Routing packets from source nodes to destination nodes in Wireless Sensor Network (WSN) is complicated due to the heterogeneous nature and distribution of sensor nodes. Packet loss problem in WSN may occur when the sensor node carrying more packets than its capacity. This can affect throughput, energy consumption and success rate of the WSN system. This paper proposes an improved Ant Colony Optimization (ACO) algorithm for packet routing to solve packet loss problem in wireless sensor network. The proposed algorithm is inspired from a variant of ACO which is Ant Colony System (ACS) that consists of local and global pheromone updates to enhance routing path exploration and exploitation. Experimental results showed that the proposed algorithm improved the performance of the proposed ACS algorithm in terms of reducing packet loss and increasing the energy efficiency of sensor nodes.
\end{abstract}

Keywords: Wireless sensor network, packet routing, packet loss, ant colony system

\section{Introduction}

Wireless sensor network (WSN) is a large-scale networking area that consists of geographically distributed sensor nodes with limited capabilities, such as storage, battery power, and communication capabilities [1]. Sensor nodes are responsible in sensing any changes in the WSN environment, communicate between the other sensor nodes during packets transmission, and sometimes perform basic computation operation with the collected data. WSN has been applied in many real applications such as healthcare [2, 3], military [4], environment monitoring [5, 6] and industrial [7]. Sensor nodes that consist of source and destination nodes are geographically placed by using static, dynamic and mobile modes depending on its usage and functionalities [8].

Scalability and robustness are some of the main aspects considered in the implementation of any routing algorithm in the WSN system [9]. Due to unlimited transmission range, sensor nodes act as an intermediate medium in WSN to forward packets from source to destination nodes. Packets in WSN are sent using multi-hop technique between available sensor nodes and connected to the end user by using the network gateway. The main factors that need to be considered in selecting the next sensor node are the remaining energy of the sensor node and length to the destination node. Packet routing in WSN aims to minimize packet loss, overload, delay, and energy consumption of sensor nodes [10]. At the same time, a good routing algorithm influences the maximizing of throughput and network lifetime.

Received (July 26, 2017), Review Result (November 15, 2017), Accepted (November 26, 2017) 
Packet loss is a condition where transmitted packets from source node fail to reach at the destination node. This problem happens in WSN due to network congestion when certain sensor nodes are over utilized to handle multiple packets routing. The packet loss problem is considered critical and needs to be effectively tackled to increase the quality of throughput and success rate of the routing process [11].

This paper presents an Ant Colony System (ACS) based packet routing algorithm in WSN. ACS is a variant of Ant Colony Optimization (ACO) algorithm that uses heuristic function to construct routing solution in dynamic distributed environment [12]. Section 2 describes previous works based on ACO in WSN and the proposed algorithm is presented in Section 3. Section 4 covers experimental result and conclusion is highlighted in Section 5 .

\section{Related Works on ACO in Wireless Sensor Network}

The ACO algorithm is inspired by the foraging behavior of ants where they work in groups in finding the shortest path to food source [13]. A chemical substance called pheromone will be used as an indicator to mark the selected path. Pheromone is deposited by ants on the ground during their journey to food source from nest and vice versa. The optimal path or shortest path will be determined by the following ants based on the amount of the pheromone deposited by previous ants. The shortest path that has high pheromone value is better than the path with low pheromone value [14]. This cooperative communication behavior and the other unique characteristics make the ant-based algorithm as a promising model in building a new algorithm.

Various types of the ACO algorithm have been proposed by researchers such as Ant System (AS), ACS, Elitist Ant System (EAS), Max-Min Ant System (MMAS), and RankBased Ant System (RAS) [13]. ACO has successfully solved many problems such as travelling salesman problem [15], fault tolerance [16], sequential ordering [17], and grid scheduling [18]. ACO has recently been applied by researchers to solve packet routing problem in WSN because of its adaptability on static, dynamic, and mobile WSN environments [19].

The Energy Efficient Ant-Based Routing (EEABR) algorithm was proposed by [20] with the objective to minimize the communication overload and energy consumption of sensor nodes. Probabilistic decision rule is used in the sensor node selection process and global pheromone update is implemented to increase the pheromone on selected path to encourage its attractiveness in the next iteration. An experiment was conducted to analyze the performance of EEABR with Basic Ant Based Routing (BABR) algorithm and Improved Ant Based Routing (IABR) algorithm in terms of energy efficiency. Despite EEABR having better performance, it did not effectively consider the exploration of alternative routing path that can avoid the dead node problem which affect the network lifetime of the system.

[21] proposed a routing algorithm based on MMAS to solve delay, packet loss, and energy consumption problem in WSN. The search angle technique has been proposed by the algorithm to limit the search area of ants during the sensor node selection process. Ants can increase the speed to search for potential sensor nodes while nodes can only broadcast their information to the neighbors within the search angle area. This approach is claimed to reduce the energy consumption of sensor nodes. The amount of pheromone on each sensor node is different, where sensor nodes on the optimal path will get more pheromone as compared to the others. In order to control the stagnation in WSN, the quantity of pheromone is limited to the maximum and minimum ranges based on the MMAS concept. The experimental results showed that the proposed algorithm has lower packet loss, dead node, and delay as compared to the basic MMAS algorithm. Despite its performance, the exploration of new search areas and the pheromone update technique were not successful in preventing the hotspot problem in WSN. 
[22] proposed a routing algorithm that incorporates the mobile sink technique into the ACO algorithm to improve the network lifetime of dynamic WSN. The responsibility of ants in the proposed algorithm is to calculate the energy level of each sensor node while selecting the best location to allocate the mobile sink node. The location of the mobile sink node changes based on the energy level of sensor nodes. Packets will be forwarded to the mobile sink node by sensor nodes with high energy level through the multi-hop technique. The approach of the mobile sink node is expected to reduce the number of hops taken by ants to forward packets to the destination, and save the energy of sensor nodes. It was proven that the proposed algorithm is more effective in the mobile WSN environment as compared to the static WSN environment. However, ants in the proposed algorithm will select the same path in submitting packets because there is no pheromone update function that can encourage the exploration of new search areas. This may lead to the hotspot problem where certain sensor nodes drastically drain their energy.

[23] proposed a hybrid routing algorithm that integrates the cluster technique and ACO algorithm with the aim to improve the energy efficiency and network lifetime of sensor nodes in WSN. The best sensor node in each cluster will be selected as a cluster head based on residual energy and distance from the sensor node to the destination node. Meanwhile, the ACO algorithm is used in finding the optimal path between the cluster head and neighbor node. To prevent stagnation, the pheromone update technique is applied on visited paths by the ACO algorithm. The proposed algorithm was evaluated against Low Energy Adaptive Clustering Hierarchy (LEACH) and PARA algorithms in terms of energy consumption and number of survival nodes. Even though the results showed that the proposed algorithm is favorable in both aspects, the validation process did not cover the delay and throughput.

\section{Enhanced Ant Colony System for Packet Routing in Wireless Sensor Network}

Enhanced Ant Colony System (EACS) algorithm is proposed to enhance the routing process in WSN in terms of increasing energy efficiency and reducing packet loss. The proposed algorithm is based on the ACS algorithm that consists of probabilistic decision rule, local pheromone update, and global pheromone update. There are two types of ants which are forward ant and backward ant. The forward ant is created at the source node to construct routing path to the destination node. The main task for the forward ant is to construct a path by moving from one sensor node to the other sensor nodes using multihop technique before forwarding the data packets. The information of every visited sensor node is saved in the ant's memory. A routing table on each sensor node that contains current pheromone value and energy level of node will be initialized so that the ant will choose the best sensor node using probabilistic decision rule. This approach encourages the selection of edges that leads to the highest energy sensor node.

The local pheromone update is applied by the forward ant on the last traversed edge before destination node to reduce the pheromone intensity on this edge while encouraging the use of alternative edge to the destination node. This approach can also reduce the hotspot problem where sensor nodes near the destination node will drastically drain their energy as compared to the other sensor nodes further away [10], [24]. This problem happens because sensor nodes near the destination node consume more energy to forward packets on behalf of the other sensor nodes located far away from the destination node. To overcome this problem, the local pheromone update is introduced to reduce the pheromone of the last visited node before the destination node. The proposed local pheromone update is defined by:

$$
\tau_{(r, s)}=(1-\varphi) *\left(\tau_{(r, s)}\right)-\frac{E_{r}}{E_{i}}
$$


where $\tau_{(r, s)}$ is the current pheromone value of the edge, $\varphi$ is the coefficient value that can control the range of pheromone values, $E_{i}$ is the initial energy of sensor node, and $E_{r}$ is the remaining energy of sensor node. The forward ant will be transformed into a backward ant once it reaches the destination node. The backward ant is responsible to perform global pheromone update to all previously traversed sensor nodes through backward movement to increase the pheromone value so that the path becomes attractive to the following ants. The number of visited nodes and amount of energy used are considered in the calculation of the global pheromone update formula. The attraction to the optimal path by the following ants can be increased through the global pheromone update. The global pheromone update is adopted from [13] and the formula is defined by:

$$
\sigma_{(r, s)}=(1-\rho) * \tau_{(r, s)}+\left(\Delta \tau_{(r, s)}\right)
$$

where $\rho$ is the evaporation rate value and $\Delta \tau_{(r, s)}$ is defined by the following formula:

$$
\Delta \tau_{(r, s)}=\frac{E_{a v g}}{N_{r}}
$$

where $E_{\text {avg }}$ is the average energy of visited sensor nodes and $N_{r}$ is the number of visited nodes from node $r$ until the destination node.

\section{Experimental Result}

Experiments were conducted to compare the performance of the proposed EACS algorithm with EEABR algorithm and Cost-aware Ant Routing (SC) algorithm in terms of packet loss rate, latency, and energy efficiency. All the experiments were performed using 9, 25, 49, and 100 sensor nodes and simulated for 100 seconds. The same simulation parameters used by [19] have been adopted except for the number of nodes as shown in Table 1. In this experiment, the source node and destination node are set to be in static mode. The source rate is the number of packets sent per second while the destination rate is the number of packets requested per second by the destination node. Constant Bit Rate (CBR) is used as data traffic in this experiment, where the static amount of bandwidth is supplied during the simulation process. The speed of transferring packets is set to $250 \mathrm{kbps}$.

Table 1. Simulation Parameters

\begin{tabular}{|l|l|}
\hline Parameter & Value \\
\hline Number of Nodes & $9,25,49,100$ \\
\hline Source Type, Radius, Rate & Static, Random, 1, 4 \\
\hline Destination Type, Radius, Rate & Static, Random, 1, 0.5 \\
\hline Data Traffic & Constant Bit Rate (CBR) \\
\hline Data Rate & 250 Kbps \\
\hline Simulation Time & 100 seconds \\
\hline Nodes Energy & 30 Joules \\
\hline
\end{tabular}

Packet loss is a condition where submitted packet fails to reach the destination node. Figure 1 shows the results of packet loss rate throughout the simulation time. EACS has the lowest packet loss rate compared to EEABR and SC when using 25 nodes, 49 nodes, and 100 nodes. In EACS, the application of local pheromone update encourages exploration to other nodes near the destination node to reduce energy depletion. In addition to that, the use of global pheromone update improves the exploration of optimal 
path from source to destination node. Improvement to both exploration and exploitation would definitely reduce the packet loss rate.

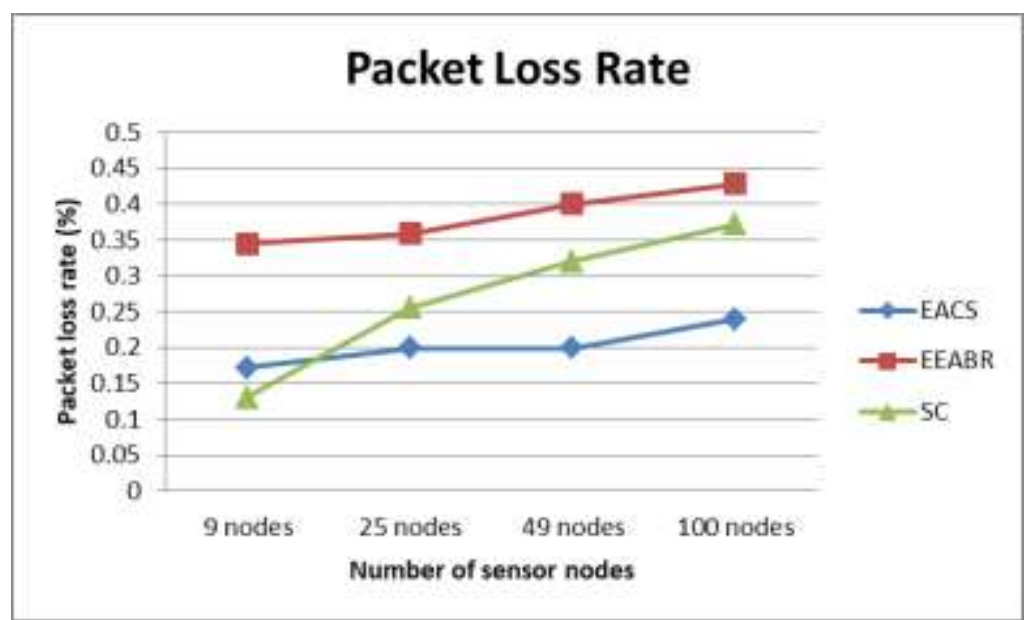

Figure 1. Comparison of Packet Loss Rate between Algorithms

Latency refers to the delay from initial packet submission at the source node to the packet arrival at the destination node. Figure 2 shows the latency value of algorithms where the proposed EACS algorithm has the lowest and constant value when using 25 nodes, 49 nodes, and 100 nodes. In EACS, routing information such as pheromone value and remaining energy of each sensor node are stored in the routing table and can be referred by the following ants to construct the routing path. This approach can save a lot of time in constructing a new path to route the following packets.

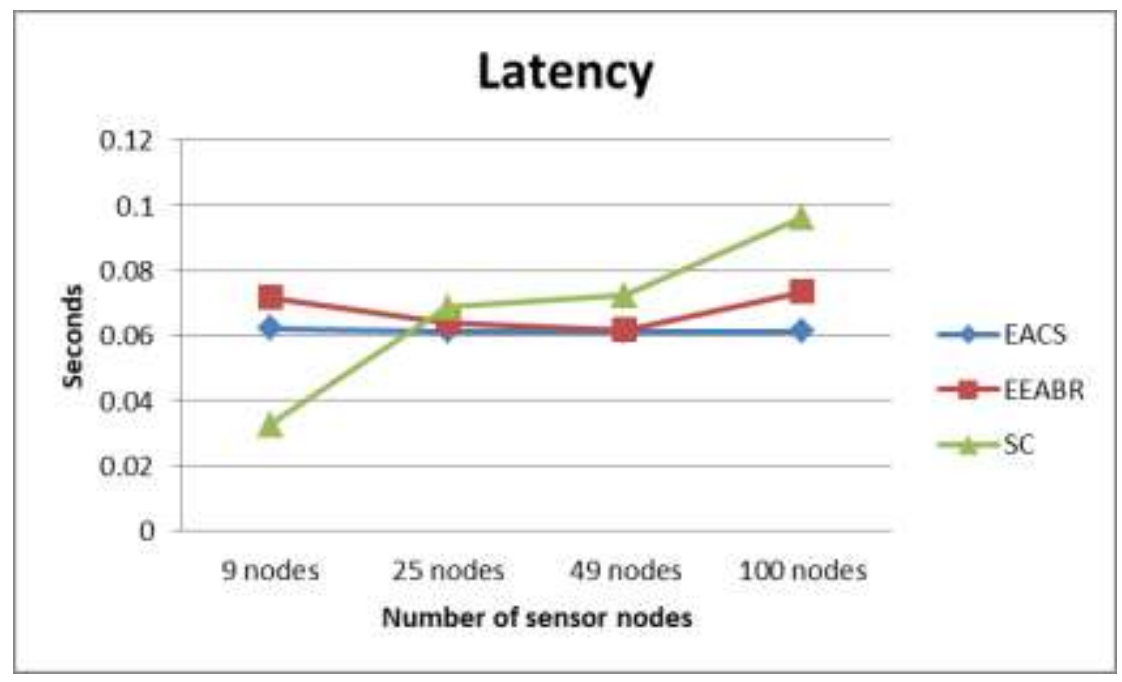

Figure 2. Comparison of Latency between Algorithms

Figure 3 depicts the results of energy efficiency. Energy efficiency is measured by the total number of packets received by the destination node per total energy used by all sensor nodes. Good energy efficiency is expected to prolong the network lifetime of the system through reduction of power consumption in sensor nodes. Lower packet loss rate and energy consumption for EACS has resulted in the highest energy efficiency compared to EEABR and SC when routing packets using 25 nodes, 49 nodes, and 100 nodes. 


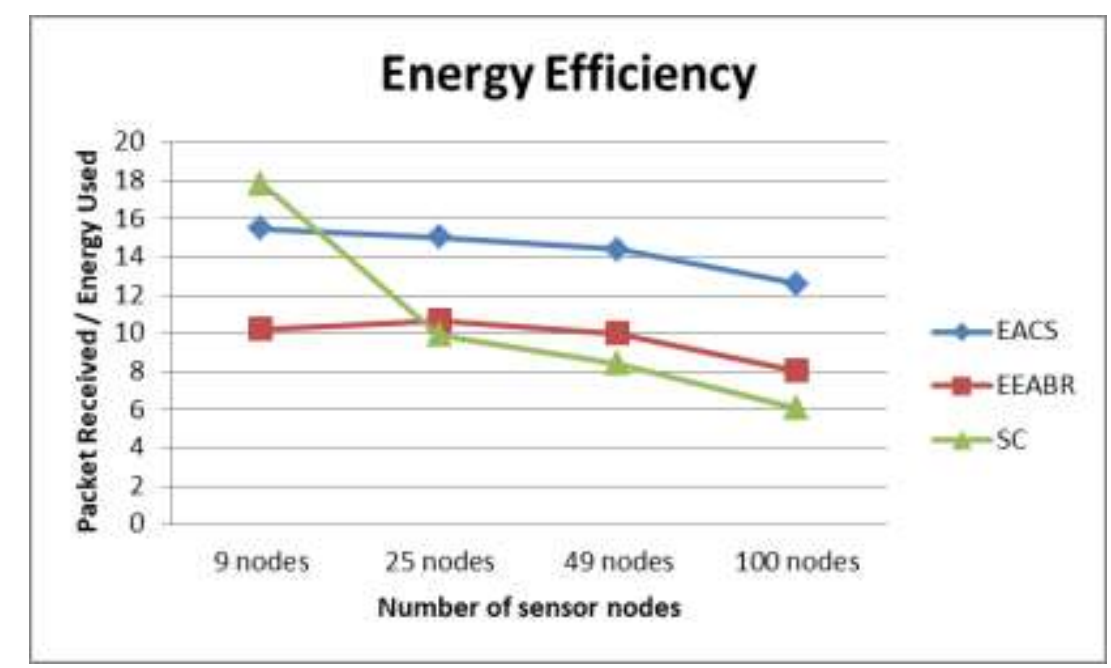

Figure 3. Comparison of Energy Efficiency between Algorithms

\section{Conclusion}

The proposed EACS algorithm is based on the ACS technique that combines the probabilistic decision rule, local pheromone update, and global pheromone update. The probabilistic decision rule is an important part in selecting the best sensor nodes to forward packets while considering the pheromone value and energy level of sensor nodes. The local pheromone update is used by EACS to prevent the hotspot problem by encouraging the exploration of other potential paths during the simulation process. In order to influence the following ants to select the optimal path, the global pheromone update is applied to increase the pheromone value on the selected path. The proposed EACS algorithm can increase the network lifetime of WSN by distributing packets to several potential optimal paths in balancing the energy level of sensor nodes. The proposed algorithm is promising to be implemented in static WSN system. For future works, it is essential to explore the possibility of using the proposed algorithm in a dynamic WSN system so that its full capability can be exploited to overcome real problems such as geographical condition and sensor lifetime.

\section{Acknowledgements}

The authors wish to thank the Ministry of Higher Education Malaysia in funding this study under the Trans Disciplinary Research Grant Scheme, S/O code 13164 and Research and Innovation Management Centre, Universiti Utara Malaysia for the administration of this study.

\section{References}

[1] L. Chhaya, P. Sharma, G. Bhagwatikar and A. Kumar, "Wireless sensor network based smart grid communications: Cyber-attacks, intrusion detection system and topology control”, Electronics, vol. 6, no. 5, (2017), pp. 1-22.

[2] P.W. Jambhulkar and V. Baporikar, "Wireless sensor network for heart disease detection using data mining technique", Int. J. Innovative Research in Computer and Communication Engineering, vol. 3, no. 6, (2015), pp. 5947-53.

[3] K. J .Kappiarukudil and M. V. Ramesh, "Real-time monitoring and detection of "heart attack" using wireless sensor networks", Fourth International Conference on Sensor Technologies and Applications; Venice, Italy, (2010).

[4] I. Ahmad, K. Shah and S. Ullah, "Military applications using wireless sensor networks: a survey", Int. J. Engineering Science, vol. 6, no. 6, (2016), pp. 7039-7043.

[5] R. K. Kumar, M. C. Mohan, S. Vengateshapandiyan, M. M. Kumar and R. Eswaran, "Solar based advanced water quality monitoring system using wireless sensor network", Int. J. Science, Engineering and Technology Research, vol. 3, no. 3, (2014), pp. 385-89. 
[6] J. J. Diaz, J. G. Colonna, R. B. Soares, C. M. S. Figueiredo and E. F. Nakamura, "Compressive sensing for efficiently collecting wildlife sounds with wireless sensor networks", 21st International Conference on Computer Communications and Networks; Munich, Germany, (2012).

[7] R. V. Arvind, R. R. Raj, R. R. Raj and N. K. Prakash, "Industrial automation using wireless sensor networks", Indian J. Science and Technology, vol. 9, no. 8, (2016), pp.1-8.

[8] H.J.A. Nasir and K.R. Ku-Mahamud, "Wireless sensor network: A bibliographical survey", Indian J. Science and Technology, vol. 9, no. 38, (2016), pp. 1-21.

[9] M.S.V. Dhage, A.N. Thakre and S.W. Mohod, "A review on scalability issue in wireless sensor networks", Int. J. of Innovative Research in Advanced Engineering, vol. 1, no. 10, (2014), pp. 463-466.

[10] B. M. M. El-Basioni, S. M. A. El-Kader, H. S. Eissa and M. M. Zahra, "An optimized energy-aware routing protocol for wireless sensor network", Egyptian Informatics J., vol. 12, no. 2, (2011), pp. 61-72.

[11] H. J. A. Nasir and K. R. Ku-Mahamud, "Ant colony optimization approaches in wireless sensor network: Performance evaluation”, J. Computer Science, vol. 13, no. 6, (2017), pp. 153-164.

[12] M. Dorigo and L. M. Gambardella, "Ant colony system: a cooperative learning approach to the traveling salesman problem”, IEEE Transactions on Evolutionary Computation, vol. 1, no. 1, (1997), pp. 53-66.

[13] M. Dorigo and T. Stützle, "Ant Colony Optimization”, MIT Press, Cambridge, (2004).

[14] Y. Liu, "Research on the algorithm optimization of improved ant colony algorithm-LSACA", Int. J. Signal Process, Image Process, and Pattern Recognition, vol. 9, no. 3, (2016), pp. 143-154.

[15] E. Lizárraga, O. Castillo and J. Soria, "A method to solve the traveling salesman problem using ant colony optimization variants with ant set partitioning", Recent Advances on Hybrid Intelligent Systems, (2013), pp. 237-246.

[16] S. Bukhari and K. R. Ku-Mahamud, "Fault tolerance grid scheduling with checkpoint based on ant colony system”, J. Computer Science, vol. 13, no. 8, (2017), pp. 363-370.

[17] R. Skinderowicz, "Population-based ant colony optimization for sequential ordering problem", in Computational Collective Intelligence, Springer, Cham, (2015), pp. 99-109.

[18] H. J. A. Nasir and K.R. Ku-Mahamud, "Grid load balancing using ant colony optimization", 2010 2nd International Conference on Computer and Network Technology, Bangkok, Thailand, (2010).

[19] A. M. Zungeru, L. M. Ang and K. P. Seng, "Termite-hill: performance optimized swarm intelligence based routing algorithm for wireless sensor networks", J. Network and Computer Application, vol. 35, no. 6, (2012), pp. 1901-1917.

[20] T. Camilo, C. Carreto, J. S. Silva and F. Boavida, "An energy-efficient ant-based routing algorithm for wireless sensor networks", International Workshop on Ant Colony Optimization and Swarm Intelligence, Brussels, Belgium, (2006).

[21] L. Luo and L. Li, "An ant colony system based routing algorithm for wireless sensor network", 2012 International Conference on Computer Science and Electronics Engineering, Hangzhou, China, vol. 2, (2012).

[22] A. Singh and S. Behal, "Ant colony optimization for improving network lifetime in wireless sensor networks", Int. J. Engineering Sciences, vol. June 2013, no. 8, (2013), pp. 1-12.

[23] Y. C. Rao and S. Rani, "Energy efficiency and maximizing network lifetime for WSNs using ACO algorithm", Int. J. Innovative Technology and Exploring Engineering, vol. 5, no. 2, (2015), pp. 15-20.

[24] J.-Y. Kim, T. Sharma, B. Kumar, G.S. Tomar, K. Berry and W.-H. Lee, "Intercluster Ant Colony Optimization Algorithm for Wireless Sensor Network in Dense Environment”, Hindawi International Journal of Distributed Sensor Network, vol. 2014, (2014), pp.1-8.

[25] T. Sharma, B. Kumar and G.S. Tomar, "OCEA: An Optimal Cluster Energy Aware Routing Protocol for Wireless Sensor Networks", International Journal of Emerging Sciences., vol.3, no. 1, (2013), pp95107.

\section{Authors}

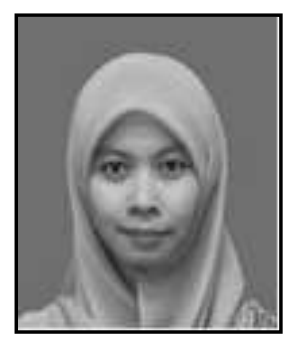

Husna Jamal Abdul Nasir, she holds a Bachelor and Masters Degree in Information Technology from Universiti Utara Malaysia, Malaysia in 2009 and 2011 respectively. She is currently a $\mathrm{PhD}$ student in Computer Science at Universiti Utara Malaysia. Her doctoral work is about wireless sensor network. As an academic, her research interests include computer network and intelligent agent. 


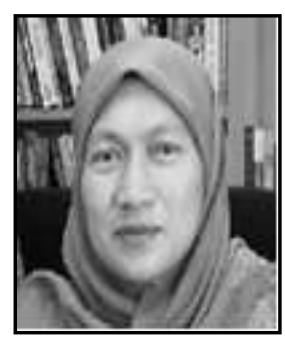

Ku Ruhana, she holds a Bachelor in Mathematical Sciences and a Masters Degree in Computing, both from Bradford University, United Kingdom in 1983 and 1986 respectively. Her $\mathrm{PhD}$ in Computer Science was obtained from Universiti Pertanian Malaysia in 1994. As an academic, her research interests include computer systems performance modeling, ant colony optimization and intelligent agent.

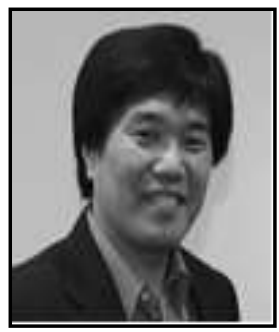

Eiji Kamioka, he is a Professor at Shibaura Institute of Technology. He received his B.S., M.S. and D.S degrees in Physics from Aoyama Gakuin University, Japan. Before joining SIT, he was working for SHARP Communications Laboratory, Institute of Space and Astronautical Science (ISAS) as a JSPS Research Fellow and National Institute of Informatics (NII) as an Assistant Professor. His current research interests encompass mobile multimedia communications and ubiquitous computing. 